\title{
Generalized Den Hartog tuned mass damper system for control of vibrations in structures
}

\author{
I. M. Abubakar \& B. J. M. Farid \\ Department of Civil Engineering, Faculty of Engineering, \\ Altahadi University, Sirte, Libya
}

\begin{abstract}
The Den Hartog method is one of old, but efficient methods for the design of tuned mass damper (TMD) systems for passive control vibrations in buildings subjected to earthquake excitations. However, Den Hartog, in his model, did not include the main mass (structure) damping in deriving the equations for computing the optimum design parameters for the TMD system (damping and frequency ratios). In this work an attempt is made to generalize the Den Hartog method to include the effect of the structure damping in the derived equations. The dynamic equations for a TMD system attached to SDOF structure with damping are derived. Then, using the complex numbers technique, the equation for maximum displacement of the structure when subjected to harmonic excitation is derived. The numerical optimization technique is used to compute the optimum values of TMD parameters that will minimize the maximum displacement of the structure. Finally, the curve fitting procedure with trial functions is used to obtain equations for computing the optimum frequency ratio and optimum damping ratio for a TMD system.
\end{abstract}

Keywords: tuned mass damper, Den Hartog model, structural damping, earthquake engineering, harmonic excitation.

\section{Introduction}

The tuned mass damper (TMD) system is a passive energy absorbing device consisting of a translational mass, translational spring and translational viscous damper attached to a vibrating structure to reduce undesirable vibrations. There has been a considerable amount of research done on how best to design the mass dampers for use in the passive control of structures under excitation forces such 
as wind and earthquake. Most of the researchers agreed that the performance of TMDs is sensitive to the accuracy of tuning the natural frequency of the TMD to the natural frequency of the structure. Den Hartog [1] has derived the formula for the optimum values of the TMD parameters for an undamped SDOF structure subjected to a harmonic excitation. Warburton and Ayorinde [2] and Warburton [3] derived closed form expressions for obtaining TMD parameters for an undamped SDOF system for base harmonic and white noise random excitation. Tsai and Lin [4] studied a SDOF with TMD system where a damping in the main mass is considered with harmonic excitations at the base. Rana and Soong [5] in their paper presented a numerical study for the performance of TMD systems. Sadek et al. [6] suggested a method for estimating the design parameters of TMDs attached to a damped SDOF structure for seismic applications, the criterion used to obtain the optimum parameters was to select, for a given mass ratio, the frequency and damping ratios that would result in equal and large modal damping in the first two modes of vibration. Abubakar [7] proposed a Translational-Rotational TMD system including the structure damping.

In the present work, the Den Hartog optimization procedure for the TMD parameters with harmonic loading applied to an undamped SDOF structure is extended to consider the damping of the main structure.

\section{Mathematical model of the problem}

Figure 1 shows the model used in the present work. It consists of a SDOF structure with properties $m_{1}, k_{1}$ and $c_{1}$. The structure is attached to a TMD system with properties $m_{2}, k_{2}$ and $c_{2}$. The resulting system is two degrees of freedom system. This model is similar to the Den Hartog Model except that Den Hartog neglected the structure damping (i.e. $c_{1}=0$ ) in his study. When this system is subjected to dynamic forces $F_{1}(\mathrm{t})$ and $F_{2}(\mathrm{t})$ acting on the structure and TMD respectively, as shown in Figure 1, the dynamic equilibrium of the two masses can be represented by the following differential equations:

For mass $m_{1}$ :

$$
\begin{aligned}
& m_{1} \ddot{x}_{1}+c_{1} \dot{x}_{1}+k_{1} x_{1}-k_{2}\left(x_{2}-x_{1}\right)-c_{2}\left(\dot{x}_{2}-\dot{x}_{1}\right)=F_{1}(t) \\
& \text { or } \\
& m_{1} \ddot{x}_{1}+\left(c_{1}+c_{2}\right) \dot{x}_{1}+\left(k_{1}+k_{2}\right) x_{1}-c_{2} \dot{x}_{2}-k_{2} x_{2}=F_{1}(t)
\end{aligned}
$$

For mass $m_{2}$ :

$$
\begin{aligned}
& m_{2} \ddot{x}_{2}+c_{2}\left(\dot{x}_{2}-\dot{x}_{1}\right)+k_{2}\left(x_{2}-x_{1}\right)=F_{2}(t) \\
& \text { or } \\
& m_{2} \ddot{x}_{2}+c_{2} \dot{x}_{2}+k_{2} x_{2}-c_{2} \dot{x}_{1}-k_{2} x_{1}=F_{2}(t)
\end{aligned}
$$




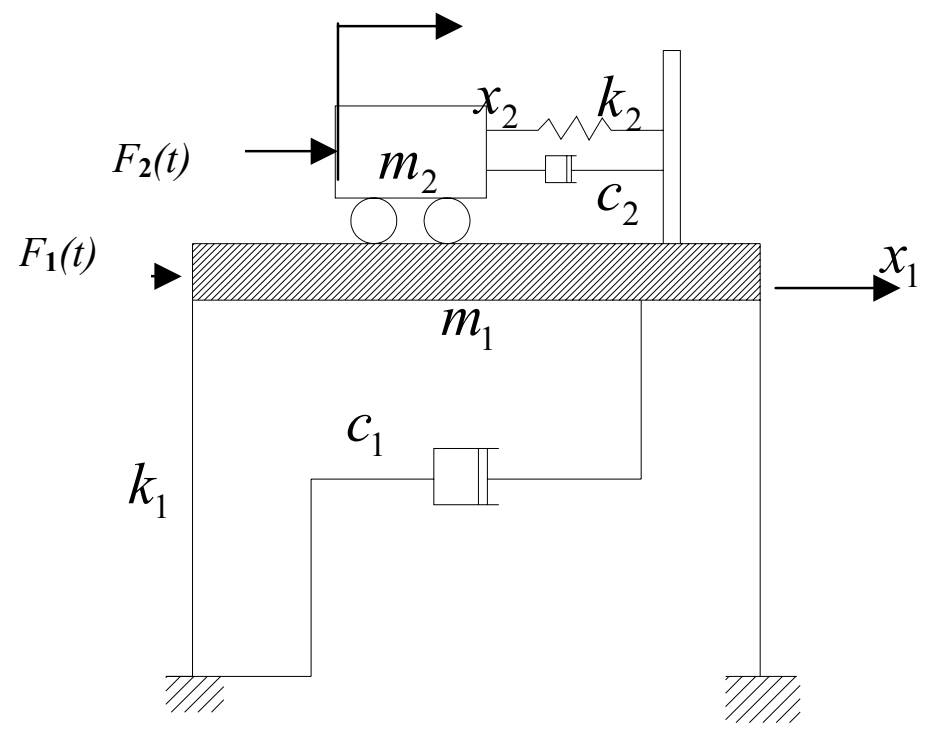

Figure 1: $\quad$ A TMD attached to SDOF structure.

The above two equations can be written in a matrix form as follows:

$$
\begin{aligned}
& {\left[\begin{array}{cc}
m_{1} & 0 \\
0 & m_{2}
\end{array}\right]\left\{\begin{array}{l}
\ddot{x}_{1} \\
\ddot{x}_{2}
\end{array}\right\}+\left[\begin{array}{cc}
c_{1}+c_{2} & -c_{2} \\
-c_{2} & c_{2}
\end{array}\right]\left\{\begin{array}{l}
\dot{x}_{1} \\
\dot{x}_{2}
\end{array}\right\}} \\
& +\left[\begin{array}{cc}
k_{1}+k_{2} & -k_{2} \\
-k_{2} & k_{2}
\end{array}\right]\left\{\begin{array}{l}
x_{1} \\
x_{2}
\end{array}\right\}=\left\{\begin{array}{c}
F_{1}(t) \\
F_{2}(t)
\end{array}\right\}
\end{aligned}
$$

Now consider the problem when the structure is subjected to harmonic force given in complex form as $F_{l}(t)=F_{o} e^{i \omega t}$ where $\omega$ is the frequency of the harmonic excitation force, and $F_{2}(t)=0$ (no load applied on the TMD). Then, equation (3) can be written as:

$$
\begin{aligned}
& {\left[\begin{array}{cc}
m_{1} & 0 \\
0 & m_{2}
\end{array}\right]\left\{\begin{array}{l}
\ddot{x}_{1} \\
\ddot{x}_{2}
\end{array}\right\}+\left[\begin{array}{cc}
c_{1}+c_{2} & -c_{2} \\
-c_{2} & c_{2}
\end{array}\right]\left\{\begin{array}{l}
\dot{x}_{1} \\
\dot{x}_{2}
\end{array}\right\}} \\
& +\left[\begin{array}{cc}
k_{1}+k_{2} & -k_{2} \\
-k_{2} & k_{2}
\end{array}\right]\left\{\begin{array}{l}
x_{1} \\
x_{2}
\end{array}\right\}=\left\{\begin{array}{c}
F_{o} e^{i \omega t} \\
0
\end{array}\right\}
\end{aligned}
$$

The solution of the problem can be obtained using complex numbers technique and assuming the responses are in the form of harmonic functions as follows: 
188 Earthquake Resistant Engineering Structures VII

$$
\begin{aligned}
& \left\{\begin{array}{l}
x_{1}(t) \\
x_{2}(t)
\end{array}\right\}=\left\{\begin{array}{l}
x_{1} \\
x_{2}
\end{array}\right\} e^{i \omega t} \\
& \left\{\begin{array}{l}
\dot{x}_{1}(t) \\
\dot{x}_{2}(t)
\end{array}\right\}=i \omega\left\{\begin{array}{l}
x_{1} \\
x_{2}
\end{array}\right\} e^{i \omega t} \\
& \left\{\begin{array}{l}
\ddot{x}_{1}(t) \\
\ddot{x}_{2}(t)
\end{array}\right\}=-\omega^{2}\left\{\begin{array}{l}
x_{1} \\
x_{2}
\end{array}\right\} e^{i \omega t}
\end{aligned}
$$

Substituting the above relations into equation (4) yields

$$
\begin{gathered}
-\omega^{2}\left[\begin{array}{cc}
m_{1} & 0 \\
0 & m_{2}
\end{array}\right]\left\{\begin{array}{l}
x_{1} \\
x_{2}
\end{array}\right\} e^{i \omega t}+i \omega\left[\begin{array}{cc}
c_{1}+c_{2} & -c_{2} \\
-c_{2} & c_{2}
\end{array}\right]\left\{\begin{array}{l}
x_{1} \\
x_{2}
\end{array}\right\} e^{i \omega t} \\
+\left[\begin{array}{cc}
k_{1}+k_{2} & -k_{2} \\
-k_{2} & k_{2}
\end{array}\right]\left\{\begin{array}{l}
x_{1} \\
x_{2}
\end{array}\right\} e^{i \omega t}=\left\{\begin{array}{l}
F_{0} \\
0
\end{array}\right\} e^{i \omega t}
\end{gathered}
$$

or

$$
\begin{gathered}
{\left[\begin{array}{cc}
-\omega^{2} m_{1}+i \omega\left(c_{1}+c_{2}\right)+\left(k_{1}+k_{2}\right) & -i \omega c_{2}-k_{2} \\
-i \omega c_{2}-k_{2} & -\omega^{2} m_{2}+i \omega c_{2}+k_{2}
\end{array}\right]\left\{\begin{array}{l}
x_{1} \\
x_{2}
\end{array}\right\}} \\
=\left\{\begin{array}{c}
F_{0} \\
0
\end{array}\right\}
\end{gathered}
$$

Solving for $x_{1}$ and rearranging, yields

$$
x_{1}=F_{0} \sqrt{\frac{a^{2}+b^{2}}{c^{2}+d^{2}}}
$$

where

$$
\begin{aligned}
& a=k_{2}-\omega^{2} m_{2} \quad, \quad b=\omega c_{2} \\
& c=\left[\omega^{4} m_{1} m_{2}-\omega^{2}\left\{m_{2}\left(k_{1}+k_{2}\right)+m_{1} k_{2}+c_{1} c_{2}\right\}+k_{1} k_{2}\right] \\
& d=\left[\omega\left(c_{2} k_{1}+c_{1} k_{2}\right)-\omega^{3}\left\{\left(c_{1}+c_{2}\right) m_{2}+c_{2} m_{1}\right\}\right]
\end{aligned}
$$

Defining the following non-dimensional parameters for the structure and TMD system

$$
\begin{aligned}
& \omega_{1}=\sqrt{\frac{k_{1}}{m_{1}}} \quad \text { (Natural frequency of the structure) } \\
& \xi_{1}=\frac{c_{1}}{2 \omega_{1} m_{1}} \quad \text { (Damping ratio of the structure) }
\end{aligned}
$$


$\omega_{2}=\sqrt{\frac{k_{2}}{m_{2}}} \quad$ (Natural frequency of the T-R TMD alone)

$\xi_{2}=\frac{c_{2}}{2 \omega_{2} m_{2}} \quad$ (Translational damping ratio of T-R TMD)

$q=\frac{\omega_{2}}{\omega_{1}} \quad$ (Ratio of T-R TMD frequency to structure frequency)

$\mu=\frac{m_{2}}{m_{1}} \quad$ (Ratio of the mass of the T-R TMD to the mass of the structure)

$r=\frac{\omega}{\omega_{1}} \quad$ (Ratio of exciting force frequency to the frequency of structure)

Then equation (8) can be written in non-dimensional form as follows:

$$
\bar{x}=\sqrt{\frac{\bar{a}^{2}+\bar{b}^{2}}{\bar{c}^{2}+\bar{d}^{2}}}
$$

where

$$
\begin{gathered}
\bar{a}=q^{2}-r^{2} \\
\bar{b}=2 \xi_{2}^{2} r q \\
\bar{c}=r^{4}-r^{2}\left(q^{2}(1+\mu)+1+4 \xi_{1} \xi_{2} q\right)+q^{2} \\
\bar{d}=2 r q \xi_{2}+2 r q^{2} \xi_{1}-r^{3}\left(2 \xi_{1}+2 \xi_{2} q \mu+2 \xi_{2} q\right)
\end{gathered}
$$

In the Den Hartog method, the optimum parameters of the TMD system $\left(\xi_{2}\right.$ and $q$ ) for a specific mass ratio $\mu$ and structure damping ratio $\xi_{1}$, are defined as those parameters which minimize the maximum displacement of the structure when subjected to harmonic excitation. For this purpose, a special numerical optimization algorithm using MATLAB environment [8] is developed to search for the optimum values of $\xi_{2 \text { opt }}$ and $q_{\text {opt }}$ for a given value of mass ratio $\mu$ and structure damping ratio $\xi_{1}$. The algorithm is based on varying the variables $r, q$ and $\xi_{2}$ and searching for the values that will minimize the structure displacement. This MATLAB software was then used to find $q_{\text {opt }}$ and $\xi_{2 \text { opt }}$ for values of mass ratio $\mu$ between 0.005 to 0.1 and structure damping ratio between 0.0 and 0.05 . Figure 2 shows the effect of mass ratio $\mu$ and $\xi_{1}$ on the optimum value of damping ratio $\xi_{2 \text { opt }}$ from which it is seen that $\xi_{2 \text { opt }}$ increases with the increase of $\mu$ and $\xi_{1}$. However, the amount of increase is small for higher values of $\xi_{1}$. Figure 3 shows the effect of $\mu$ and $\xi_{1}$ on the optimum value of frequency ratio $q_{\text {opt }}$. It is shown that $q_{\text {opt }}$ decreases with the increase of $\mu$ and $\xi_{1}$. Figure 4 shows the variation of the maximum nondimensional displacement of structure with the mass ratio $\mu$. For different values of $\xi_{1}$, it can be seen that maximum displacement of the structure is reduced with the increase of $\mu$ and $\xi_{1}$ as expected. 


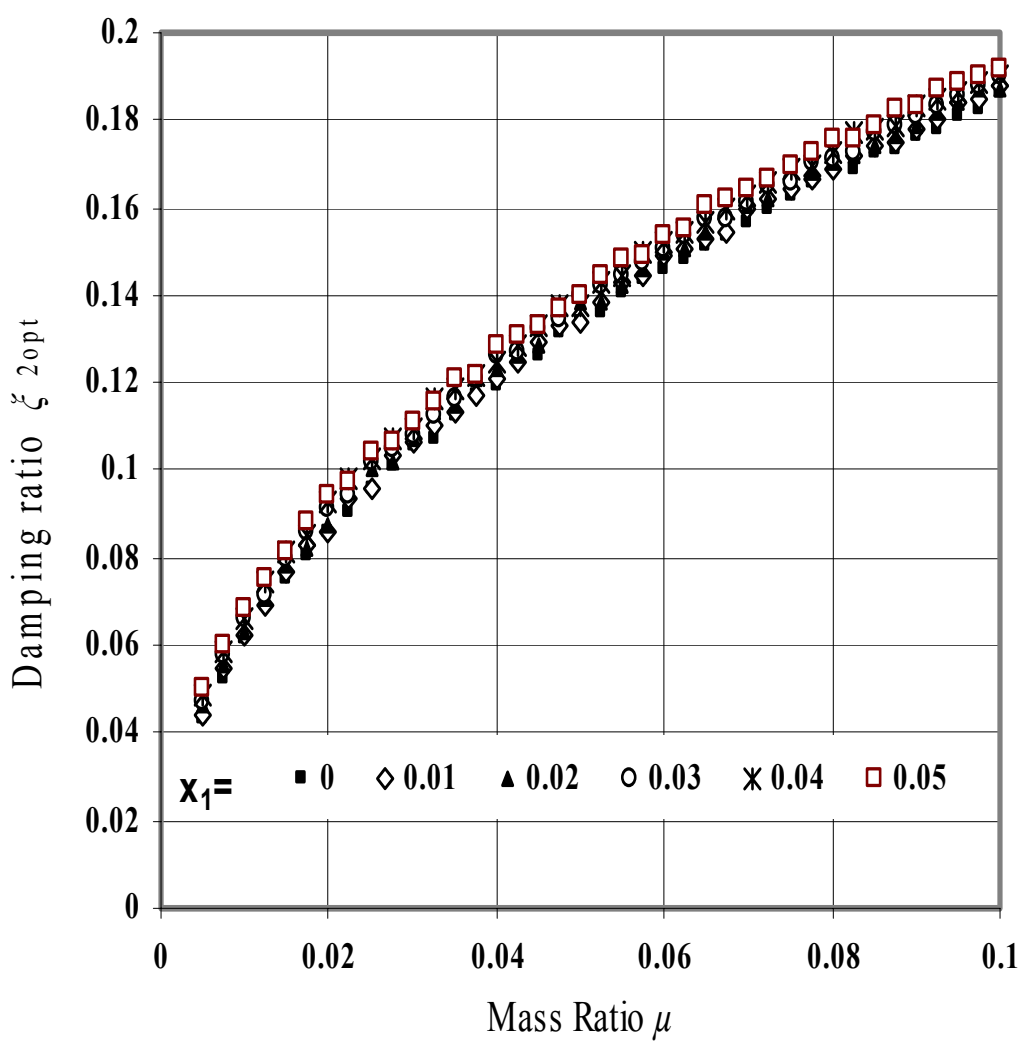

Figure 2: Variation of damping ratio $\xi_{2 \text { opt }}$ with mass ratio $\mu$ using the present study results.

\section{Practical design equations}

For Design purposes, it may be more convenient to present the optimum TMD parameters by simple design equations rather than figures. For this purpose, the curve fitting Tool Box within the MATLAB software [8] was used to carry out extensive curve fitting trials using the numerical data obtained as described above. The following suggested equations were found to give a very close approximation to the values of $\xi_{2 \text { opt }}$ and $q_{\mathrm{opt}}$ shown in figures above (curve fitting correlation coefficient was higher than 0.9994 ).

$$
\xi_{2 o p t}=\sqrt{\frac{3 \mu}{8(1+\mu)}}+\frac{0.1616 \xi_{1}}{(1+\mu)}
$$




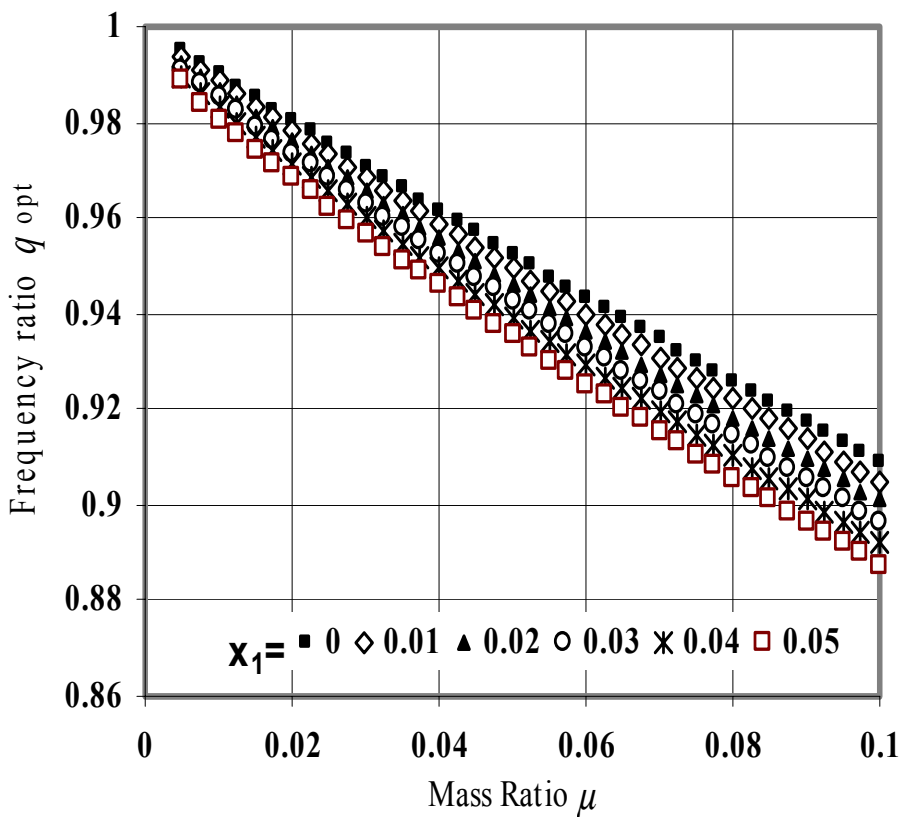

Figure 3: Variation of frequency ratio $q_{\text {opt }}$ with mass ratio $\mu$ using the present study results.

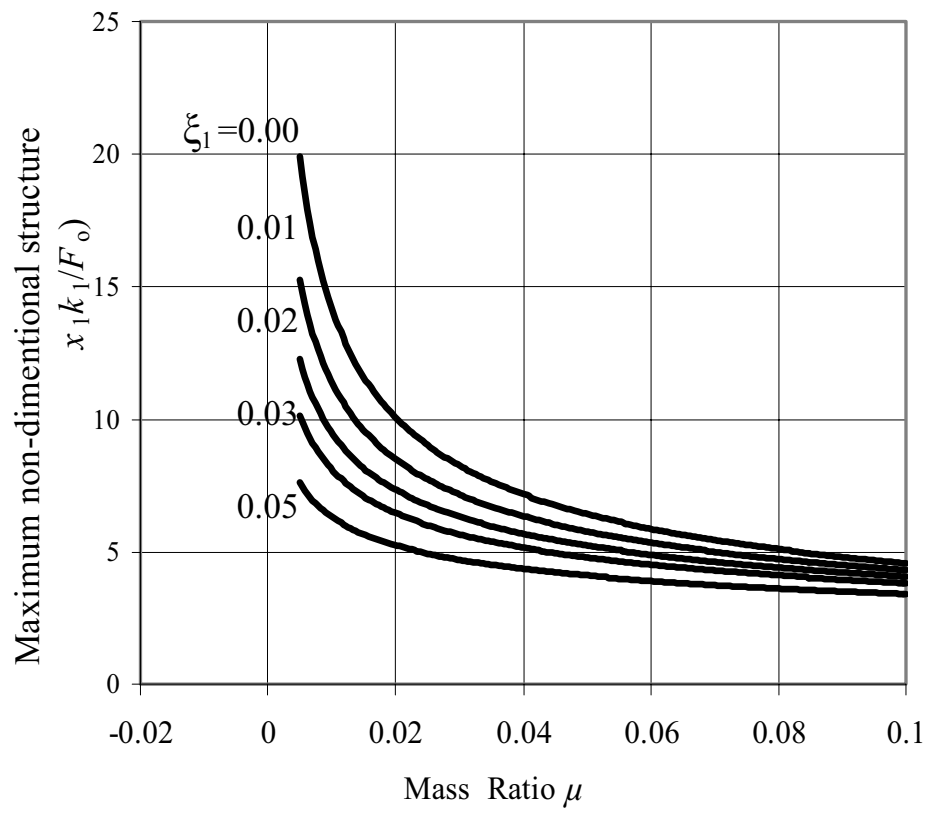

Figure 4: Variation of maximum non-dimensional structure displacement with mass ratio $\mu$ for various values of $\xi_{1}$. 
192 Earthquake Resistant Engineering Structures VII

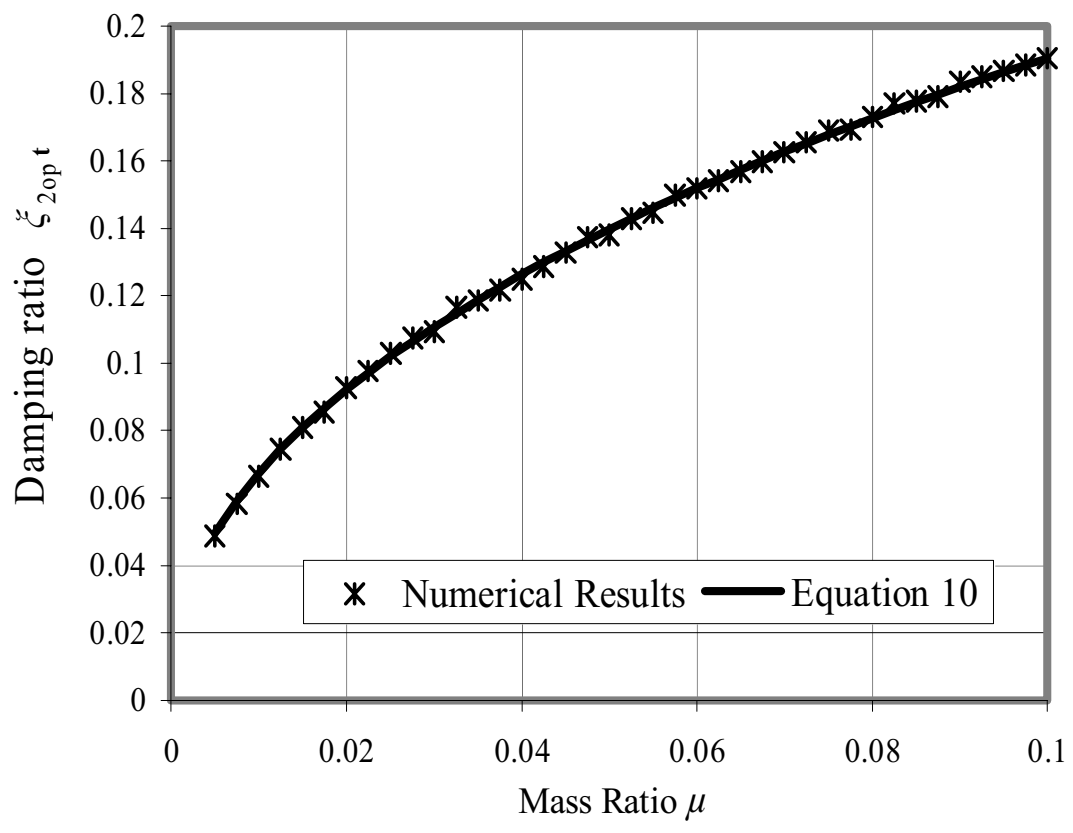

Figure 5: $\quad$ Variation of damping ratio $\xi 2_{\text {opt }}$ with mass ratio $\mu($ for $\xi 1=0.04)$.

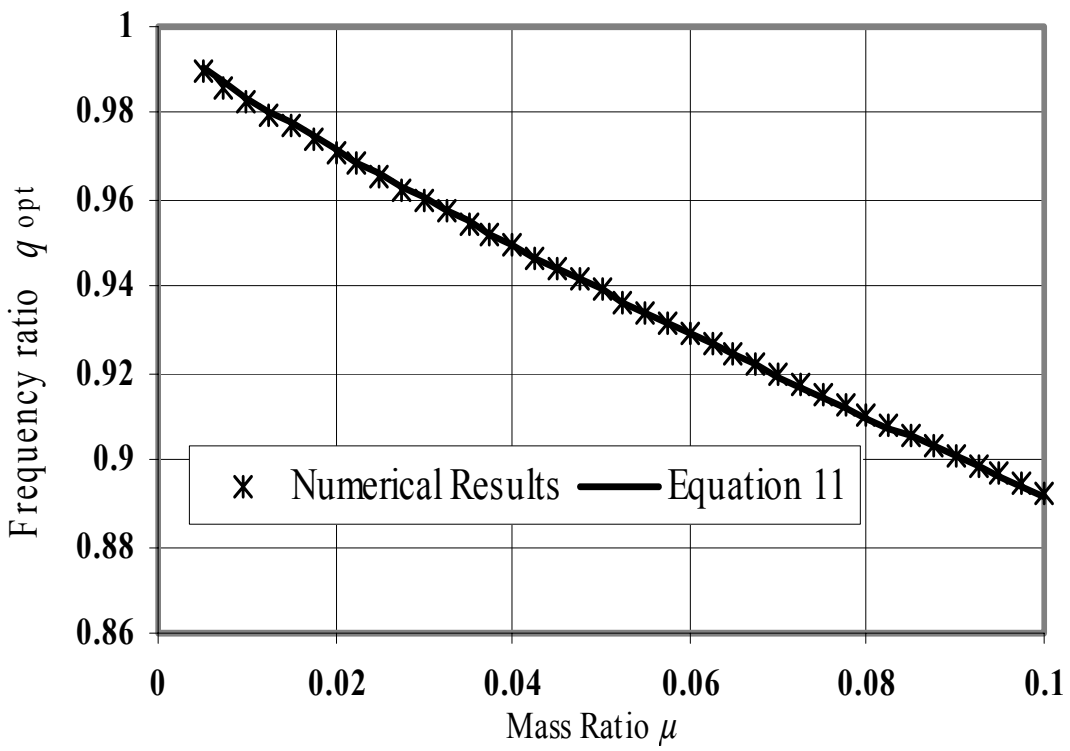

Figure 6: $\quad$ Variation of frequency ratio $q_{\mathrm{opt}}$ with mass ratio $\mu(\mathrm{x} 1=0.04)$. 


$$
q_{\text {opt }}=\left(\frac{1}{1+\mu}\right)\left(1-1.5906 \xi_{1} \sqrt{\frac{\mu}{(1+\mu)}}\right)
$$

These equations were found to give a maximum error (with respect to numerical results) of approximately 1.5 per cent in $\xi_{2 \text { opt }}$ and $q_{\text {opt }}$. It should be noted that these equations are suggested for the range of $\mu$ between 0.005 up to 0.1 . The results obtained using the above equations are compared with those of numerical analysis in Figure 5 and 6 respectively. The figures show the close agreement between the numerical results and the equations results. Equations 10 and 11 are reduced to the Den Hartog equations [1] for $\xi_{1}=0.0$.

\section{Conclusions}

The Den Hartog model for obtaining optimum design parameters for a TMD system attached to undamped SDOF structure has been extended to include the structure damping. Numerical results were obtained using numerical optimization. Finally, simple design equations were suggested for the determination of the optimum design parameters of TMD systems.

\section{References}

[1] Den Hartog, J. P. Mechanical Vibration, McGraw-Hill, New York. N.Y., 1956.

[2] Warburton, G. B. and Ayorinde, E. O., Optimum absorber parameters for simple systems, Earthq. Engng Struct. Dynam, 8, pp 197-217, 1980.

[3] Warburton, G. B., Optimal absorber parameters for various combinations of response and excitation parameters, Earthq. Engng Struct. Dynam, 10, pp 381-401, 1982.

[4] Tsai, H. C. and Lin, G. C., Optimum tuned mass dampers for minimizing steady-state response of support excited and damped systems, Earthq. Engng Struct. Dynam., 22, pp 957-973, 1993.

[5] Rahul Rana and T. T. Soong, Parametric, study and simplified design of tuned mass dampers, Engineering Structures, 20(3), pp. 193-204, 1998.

[6] Sadek, F., Mohraz, B., Taylor, A. and Chung, R., A Method of Estimating The Parameters Of Tuned Mass Dampers For Seismic Applications, Earthq. Engng Struct. Dynam, 26, pp.617-635, 1997.

[7] Abubakar, I. M., Control of RC Buildings Vibrations using Tuned Mass Dampers system, PhD Thesis Submitted To The Faculty of Engineering, Alexandria University, Alexandria, Egypt, 2006.

[8] MATLAB Version 7.10, The Language of Technical Computing, The Math Works, Inc. (2005). 